\title{
Distributed Strategic Mode Selection for Large-Scale D2D Communications Based on Queue State Information
}

\author{
Yezekael Hayel ${ }^{1,3}$, Eran Simhon ${ }^{2}$, David Starobinski ${ }^{2}$, and Quanyan $\mathrm{Zhu}^{3}$ \\ ${ }^{1}$ LIA/CERI, University of Avignon, Avignon, France, Email: yezekael.hayel@univ-avignon.fr \\ ${ }^{2}$ Division of System Engineering, Boston University, USA, Email: \{simhon,staro\}@bu.edu \\ ${ }^{3}$ Department of Electrical and Computer Engineering, New York University, USA. E-mail: \{yezekael.hayel,quanyan.zhu\}@nyu.edu.
}

\begin{abstract}
Device-to-Device (D2D) communication that enables nearby mobiles to directly communicate one with another is a new paradigm aimed at increasing the capacity of next-generation wireless networks. The coexistence of D2D and cellular communication in the same spectrum poses new challenges for resource allocations and interference management in a largescale wireless system where each mobile strategically selects its mode of communications. This paper formulates a gametheoretic framework to capture the distributed strategic behavior of a large population of mobiles in selecting their mode of communications. In particular, we investigate the impact of Queue State Information (QSI) of the base station (BS) on the mobile decisions, and we show that the common knowledge of QSI can induce bad quality of service for standard cellular traffic, when the capacity of the base station is below a certain threshold. This paradox will be used to guide the design of optimal learning and scheduling algorithms for the coexisting D2D communication networks.
\end{abstract}

\section{INTRODUCTION}

In next-generation 5G networks, Device-to-Device (D2D) communications will be incorporated into cellular networks to increase the capacity of the system by allowing direct communication between nearby mobiles. This direct communication mode will provide an efficient tool to enhance the capacity of future 5G networks. However, the coexistence of D2D and cellular communication in the same spectrum poses new challenges for resource allocations and interference management [1]. In particular, from the perspective of the Internet of Things (IoT), the increasing number of wireless devices will demand distributed solutions to meet these challenges for large-scale wireless networks.

This work aims to design efficient mechanisms to manage D2D communication inside a wireless cell without compromising the quality of service of communications. We consider inband D2D communications with underlay, i.e., communication in the licensed spectrum is used both for D2D and cellular links; D2D and cellular devices share the same radio resources. A mobile that intends to communicate with another mobile located in the same cell, can strategically determine its mode of communications, i.e. to use D2D mode or cellular mode. The D2D mode often has a lower throughput, while in contrast, communication through the base station (BS) as a relay has a higher throughput. On the other hand, D2D provides an option to circumvent congestions at the BS when a large number of mobiles choose the cellular mode.

This work aims to establish a game-theoretic framework to model the model selection problem for a large population of mobiles in the D2D communication network as depicted in Fig. 1. The decision of a mobile to select D2D mode depends on the number of mobiles who communicate using BS, and hence the Queue State Information (QSI) of the BS. With the strategic behaviors of the mobiles, the BS can determine whether to send QSI to mobiles or not in order to minimize the expected delay of cellular traffic. We leverage this framework to investigate the impact of QSI on the mobile decisions, and hence the performance of the network. We unveil a paradox whereby sharing QSI with mobile users can induce bad quality of service for standard cellular traffic, when the capacity of the base station is below a certain threshold. In other words, giving more information to users may result in worse performance when the users behave strategically. This paradox can be used to guide the design of optimal learning and scheduling algorithms for coexisting D2D and cellular communication networks.

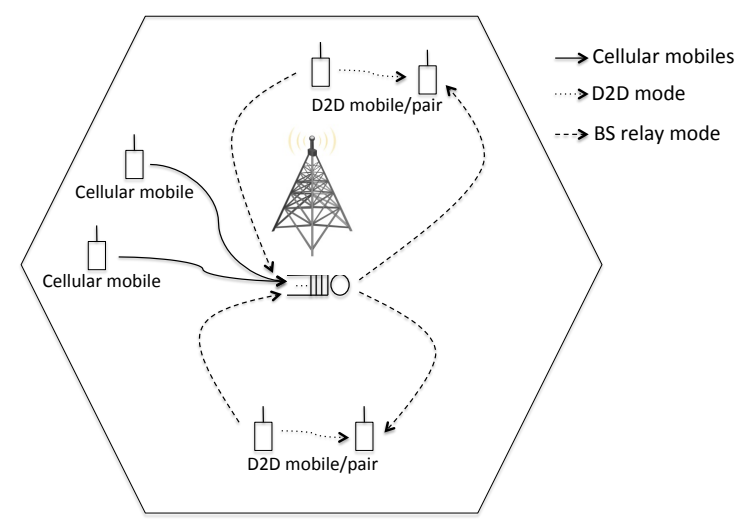

Fig. 1. Example of mode selection in a wireless cell: D2D mobiles can communicate using D2D mode or cellular mode via the BS. D2D communications via the BS will share the BS queue with other uplink cellular mobile traffic.

\section{A. Related Works}

Song et al. in [3] uses game-theoretic models to analyze non-cooperative and cooperative behaviors of mobile users in the context of D2D communication underlaying cellular networks. Mobiles can decide to use the base station as a relay instead of the D2D mode for local communication. In our work, we establish game-theoretic models for a large population of mobiles and the impact of decision making and the availability of QSI on global performance. In [4], the authors consider the problem of mode selection in D2D communications in terms of performance metrics of the queues for 
each types of mobiles (D2D and cellular mobiles). The mode selection is not determined strategically by each D2D pair, but is rather dynamically controlled by the base station according to estimated performances. Their main results are obtained using simulations. The goal of our work is to establish a theoretic foundation to provide guidelines for quantitative design of next-generation D2D communication protocols and management systems.

\section{B. Structure of the Paper}

The rest of the paper is organized as follows: Section II introduces the system models of D2D and cellular communication modes. Two cases of information structures are discussed. Subsections II-A and II-B present the scenarios without and with QSI, respectively. The optimal design of sharing or not QSI is discussed in Subsection II-C. Section III presents learning algorithms for mode selection of the mobiles. The paper is concluded in Section IV.

\section{SYSTEM MODEL}

The Queue State Information (QSI) is important for a mobile device to decide whether to communicate with its neighboring devices directly or using the base station as a relay. Direct communication usually provides inferior performance to base station relaying as D2D technologies such as Bluetooth and directWiFi [5]. Let $C_{0}$ represent this capacity limit of the D2Ds communication technology under the type of spectrum management used for direct communication in the cell (inband or outband). Besides the limited capacity, the direct communication is also constrained by the geographic distance between the transmitter and the receiver.

We model our system by considering a queueing framework as in [8]. A D2D transmitter that sends data traffic to the BS queue will interact with other D2D transmitters. Each D2D transmitter can be seen as a player in a game theoretic context; and all the players interact through a single queue system. The interdependencies of the players stem from the average delay of packets into the shared queue. As more players choose to send data via the BS queue, they experience higher average delay. Due to the large scale wireless networks, we consider indistinguishable infinitely many players as each D2D transmitter has the identical cost and action space. We define the following parameters:

- $\lambda_{D}$ Poisson rate of D2D mobile traffic,

- $\lambda_{C}$ Poisson rate of Cellular mobile traffic,

- $1 / \mu$ mean of the exponential service time,

- $C_{0}$ cost for using direct communication with the neighbor. We denote by $\bar{W}^{U}$ (resp. $\bar{W}^{O}$ ) the average sojourn time (delay) in the queue of a cellular mobile traffic when the QSI is unobservable (resp. observable) for the D2D mobiles. We consider a (First-Come-First-Serve) FCFS service policy at the BS, and each D2D mobile/pair decides wether to send or not its packets to the BS queue (mode selection), to minimize its expected cost. This type of problem, related to strategic decision in queueing systems, is known as queueing games, and surveyed in [6]. In the case of observable QSI, the concept of equilibrium is not central, as the decision of each D2D customer does not depend on the decisions of other customers but on the current state of the queue. Our study resembles that [7], which compares the outcomes of an $M / M / 1$ queue with and without QSI. But in their work, the authors compare analytically a particular case when the effective joining rates are equals in the different scenarios. This special case makes analytical comparison easier. However, our model includes D2D communication, an important feature in future $5 \mathrm{G}$ networks.

\section{A. No QSI (Unobservable System)}

In this case, the average delay $\bar{W}^{U}$ can be obtained explicitly depending on the arrival rates, the service rate and cost parameter of the queueing system. In fact, we face a standard unobservable decision strategic problem. The average delay and the proportion $p_{u}$ of D2D traffic using the BS queue are given as follows:

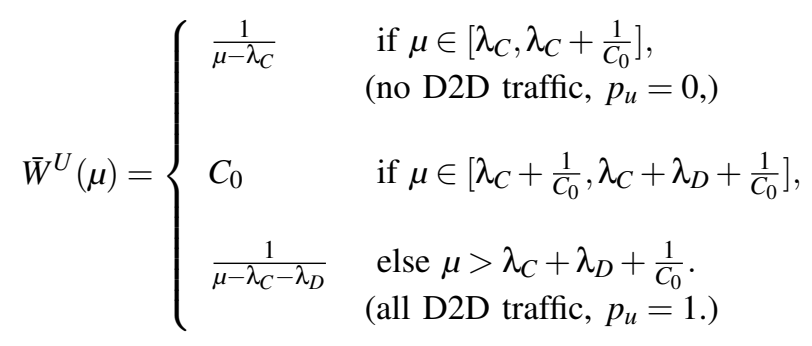

In the second case, when the equilibrium is a mixed one, the proportion of D2D mobiles that join the BS queue is explicitly given by $p_{u}=\frac{\mu-\lambda_{C}-\frac{1}{C_{0}}}{\lambda_{D}}$. Note that in this case the average delay $\bar{W}^{U}$ is equals to $C_{0}$. Indeed, all packets in the BS queue are served identically, and then have the same average sojourn time. Thus the average delay of the cellular mobile packets is the same as the D2D mobile packets into the queue. Moreover, the incoming rate $p_{u} \lambda_{D}$ of $\mathrm{D} 2 \mathrm{D}$ traffic is such that the average sojourn time is equal to $C_{0}$. This condition corresponds to the indifference principle of game theory, meaning that no individual has interest to change its decision at equilibrium. Thus, the average delay of cellular mobile traffic is also equals to $C_{0}$ at equilibrium.

\section{B. With QSI (Observable System)}

We now consider the case where D2D mobile/pairs can observe the state of the base station queue before deciding. It is shown in [8] that there exists a unique threshold $n_{s}$ such that if and only if the number of request in the queue is strictly lower than this threshold, a new packet arrival will join the BS queue. This threshold is simply given by $n_{s}=\left\lfloor\mu C_{0}\right\rfloor$. The number of packets in the BS queue is a special case of birth and death process with the transition rate $\lambda_{i}=\lambda_{C}+\lambda_{D}$ from state $i$ to $i+1$ if the state $n<n_{s}$, otherwise $\lambda_{i}=\lambda_{C}$.

The stationary probability $\pi_{i}$ for having $i$ packets in the 
queue is given by:

$$
\pi_{i}= \begin{cases}\pi_{0}\left(\frac{\lambda_{C}+\lambda_{D}}{\mu}\right)^{i} & \text { if } i \leq n_{s} \\ \pi_{0}\left(\frac{\lambda_{C}+\lambda_{D}}{\mu}\right)^{n_{s}}\left(\frac{\lambda_{C}}{\mu}\right)^{i-n_{s}} & \text { if } i>n_{s},\end{cases}
$$

with

$$
\pi_{0}=\left(\sum_{k=0}^{n_{s}}\left(\frac{\lambda_{C}+\lambda_{D}}{\mu}\right)^{k}+\frac{\lambda_{C}}{\mu+\lambda_{C}}\left(\frac{\lambda_{C}+\lambda_{D}}{\mu}\right)^{n_{s}+1}\right)^{-1} .
$$

When D2D pairs observe the QSI for their mode selection, we are able to compute the average delay $\bar{W}^{O}=\sum_{i=0}^{\infty} \pi_{i} \frac{i+1}{\mu}$.

\section{Optimal Design of the QSI}

The goal of this subsection is to address the following question: Depending on the capacity of the base station (parameter $\mu$ ), should the system provide the QSI to the D2D mobile/pairs before they make their mode selection? This question has to be answered taking into consideration the quality of service of the cellular devices, in terms of their average delay. Intuitively, to give information to strategic decision makers is always a good feature for the system where the utility function of the decision maker is the same as the provider. However, this is not the case here as we observe that it is not always beneficial to give information. We prove that it is the case when the capacity $\mu$ of the base station is lower a threshold $\mu_{0}^{*}$.

Proposition 1: There exists a threshold $\mu_{0}^{*}$ such that in order to minimize the average delay of the cellular packets in the queue, the base station should not give the queue state information (QSI) to the D2D mobiles if $\mu<\mu_{0}^{*}$. This threshold is defined as $\mu_{0}^{*}=\min \left\{\mu>0 \quad \mid \quad \bar{W}^{O}(\mu)=\bar{W}^{U}(\mu)\right\}$.

The existence of this threshold can be mathematically proven and it is depicted on a numerical example in Fig. 2. The threshold obtained in this numerical example is $\mu_{0}^{*}=5.345$.

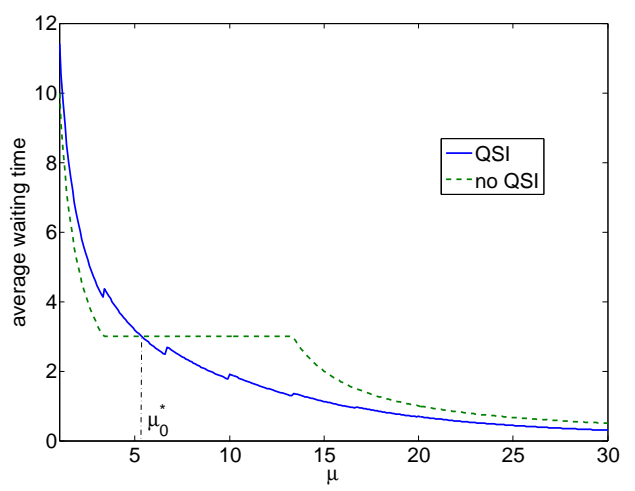

Fig. 2. Average sojourn time of cellular traffic depending on the QSI information system for D2D mobiles. The parameters are $\lambda_{C}=2, \lambda_{D}=3$ and $C_{0}=3$.

\section{LEARNING OPTIMAL STRATEGY}

In both types of information, QSI and no-QSI, the optimal decision for a D2D-pair depends on the capacity $\mu$ of the base station. In a decentralized system like the one considered here, where the D2D pairs decide selfishly to join or not the base station queue, the informations needed to take their best decision may not be known a priori. Then, learning algorithms can be used by D2D pairs to autonomously determine the best decision.

In the case of no QSI, each D2D pair can learn the probability $p(n)$ for the $n^{\text {th }}$ packet to join the BS queue using the following decision rule update:

$$
p(n+1)=p(n)+\lambda_{n}\left(\frac{1}{n} \sum_{k=1}^{n} W_{k}-C_{0}\right),
$$

where $W_{k}$ is the delay perceived by the $k^{\text {th }}$ packet sent into the base station queue. We have to consider a learning rate $\lambda_{n}$ such that this process converges when the number of packets gets large enough. A similar learning algorithm, considering QSI, has been proposed in [9]. The authors assume some uncontrolled arrivals in order to ensure that their algorithm will not stop. We do not need this assumption here as the cellular mobiles traffic is uncontrolled and join the BS queue. So, we can propose a similar learning procedure in the case of QSI. Convergence of this learning procedure is based on the theory of stochastic approximations algorithm and particularly ordinary differential equation methods [10].

\section{CONCLUSION}

In this paper, we study the impact of sharing information on the performance of cellular 5G networks with D2D users. We show that revealing the queue length to D2D customers deteriorates the quality of service for cellular users when the capacity $(\mu)$ is below a certain threshold, while it improves quality of service when the capacity is above the threshold. We plan to leverage this result to further investigate pricing schemes based on QSI and convergence of mode selection algorithms to the Nash equilibria.

\section{REFERENCES}

[1] A. Asadi, Q. Wang, V. Mancuso A Survey on Device-to-Device Communication in Cellular Networks, in IEEE Communication surveys and tutorials, vol. 16, no. 4, 2014.

[2] L. Atzori, A. Iera, and G. Morabito, The internet of things: A survey, Computer Networks, vol. 54, pp. 27872805, Oct. 2010.

[3] L. Song, D. Niyato, Z. Han, E. Hossain, Game-theoretic Resource Allocation Methods for Device-to-Device (D2D) Communication, to appear, IEEE Wireless Communications Magazine, 2014.

[4] L. Lei, X. Shen, M. Dohler, C. Lin, Z. Zhong Queuing Models with Applications to Mode Selection in Device-to-Device Communications Underlaying Cellular Networks to appear in IEEE Trans. in Wireless Communications, 2014.

[5] 3rd Generation Partnership Project, Feasibility Study for Proximity Services (ProSe), 3GPP TR 22.803 V12.2.0, Jun 2013.

[6] R. Hassin, M. Haviv, To queue or not to queue: equilibrium behavior in queueing systems, Kluwer Academic Publishers, 2002.

[7] R. Shone, V. Knight, J. Williams, Comparisons between observable and unobservable $M / M / 1$ queues with respect to optimal customer behavior, European Journal of Operational Research 227 (2013): 133-141.

[8] P. Naor, The regulation of queue size by levying tolls, Econometrica 37 (1), 1969.

[9] E. Altman, N, Shimkin, Individual equilibrium and learning in processor sharing systems,in Operations Research, 46, 6, pp. 776-784, 1998.

[10] V. Borkar Stochastic Approximation: A dynamical systems viewpoint, Cambridge University Press, 2008. 